\title{
Assessment of Cardiovascular Risk Factors among Women Who Are the Industrial Residents of Hyderabad
}

\author{
Sudha Bala ${ }^{1}$ Madhuri Taranikanti ${ }^{2}$ Aruna Kumari Yerra ${ }^{3}$ Bhavya Gundepudi ${ }^{1}$ \\ ${ }^{1}$ Department of Community Medicine, ESIC Medical College, \\ Sanathnagar, Hyderabad, India \\ 2Department of Physiology, ESIC Medical College, Sanathnagar, \\ Hyderabad, India

\begin{abstract}
Address for correspondence Sudha Bala, Assistant Professor, Department of Community Medicine, Department of Community Medicine, ESIC Medical College, Sanathnagar, Hyderabad 500038, Telangana, India (e-mail: Dr.sudhabala78@gmail.com).
\end{abstract}

${ }^{3}$ Department of Obstetrics and Gynecology, ESIC Medical College, Sanathnagar, Hyderabad, India

Ind J Car Dis Wom 2019;4:1-7

\begin{abstract}
Background and Aim Cardiovascular diseases among women are rapidly increasing as an epidemic. Identification of risk factors is very essential along with its clinical application to avoid misdiagnosis and poor management. This study aims to assess various risk factors among women who are industrial residents of Hyderabad, to estimate the risk by using Framingham risk score (FRS) categorization.

Methods A community-based cross-sectional study was undertaken among 370 women using World Health Organization (WHO) stepwise approach (STEPS) questionnaire schedule with gender-specific risk factors such as metabolic syndrome, menopause, and pregnancy-associated complications; anthropometry, blood pressure, and lipid profile measurements. Risk estimate of over 10 years has been estimated with FRS. ANOVA (analysis of variance), and chi-square test was used to depict association of risk with various variables.

Results Our study determined 3.7\% at intermediate risk and $2.5 \%$ at higher risk using FRS. Highest prevalence of risk factors included mainly poor intake of good-cholesterol diet among $96 \%$, predicted by low high-density lipoprotein (HDL) among $89 \%$, poor

Keywords

- cardiovascular diseases

- Framingham risk score

- risk factors

- metabolic syndrome intake of fruits among $81 \%$, higher waist circumference $(>80 \mathrm{~cm})$ among $66.7 \%$, raised blood glucose among $58.4 \%$, obesity (47.2\%), extra intake of salt ( $40 \%)$, and metabolic syndrome among $29.7 \%$ with predominance in younger age group.

Conclusion Estimation of cardiovascular risk at an earlier stage provides an opportunity to target young women among whom the metabolic syndrome is the major determinant with preventive strategies.
\end{abstract}

\section{Introduction}

Women play multiple roles in the household and workplace community, which might diminish their ability to engage in physical activity, may not have access to correct information about healthy diet, and access to cost-effective health promotion with early detection and treatment strategies. Gender-specific risk factors related to cardiovascular diseases (CVDs) play an important role to reduce morbidity or mortality and optimize management.

Globally, CVD is the leading cause of mortality among women. ${ }^{1}$ Further evidence supporting this has been demons- trated by national health and nutrition examination survey demonstrating a higher prevalence of myocardial infarction among women in the age group of 35 to 54 years. Women account to 242 per 100,000 population as per age-standardized deaths globally constituting to $26.7 \%$ of total deaths. Percentage of total disability-adjusted life years (DALYs) due to CVDs is approximately $12.2 \%$. In South-East Asia, deaths were approximately 274 per 100,000 women suffering from CVDs. ${ }^{2}$

As per the changing patterns, the prevalence of CVDs is increasing in India due to the epidemiologic and nutritional transition attributed to industrialization and urbanization. High epidemiologic transition-level states are found in 
Kerala, Punjab, and Tamil Nadu. Telangana belongs to lower middle epidemiologic transition level among various states contributing to DALYs loss of 3,257 per 100,000 women. ${ }^{3}$

Assessment of risk factors has been recommended by World Health Organization (WHO) stepwise approach (STEPS) for surveillance of noncommunicable diseases risk factors. These risk factors have the most significant impact on morbidity and mortality related to CVDs. This includes behavioral, metabolic, and other risk factors that are 12 in number. ${ }^{4}$ This assessment among women includes other specific factors such as menopausal status, metabolic syndrome, and pregnancy complications such as gestational diabetes and pregnancy-induced hypertension. Effects of menopause include an increase in body weight, alteration of fat distribution, centripetal obesity, and visceral fat deposition. ${ }^{5}$

There are many risk scores to predict and estimate the severity of CVDs over the coming years, such as Framingham risk score (FRS), QRISK score, WHO risk prediction chart, PROCAM, etc. FRS is the most simplified and most commonly and easily applicable that is used to estimate CVD over 10 years. This includes six coronary risk factors such as age, gender, total cholesterol (TC), high-density lipoprotein (HDL) cholesterol, smoking habits, and systolic blood pressure. ${ }^{6}$

There is a dearth of studies about the assessment of risk factors, specifically among women of Indian industrial residents in Hyderabad. Therefore, an attempt has been made to study the prevalence of risk factors of CVDs and to categorize cardiovascular risk estimate as per FRS.

\section{Material and Methods}

- Study design: Community-based cross-sectional study.

- Study area: Urban field practice area, Jeedimetla, Hyderabad, which is an industrial area of Hyderabad.

- Study period: 3 months (May 2018 to July 2018).

- Study participants: All women aged > 20 years willing to participate and residents from past 1 year are included in this study.

- Exclusion criteria: Women on hormone therapy, pregnant women as well as individuals suffering from an established case of coronary artery disease and stroke are not considered in this study.

- Sample size: Prevalence of common risk factor among urban women was found to be hypertension that is $70 \%{ }^{7}$ Taking this and calculating by formula $=4 \mathrm{pq} / \mathrm{l}^{2}$, the sample size calculated is 336 , and taking nonresponse rate of $10 \%$, the size was rounded to 370 .

- Sampling method: Simple random sampling was adopted for selecting the participants, and only one from each household was included to avoid clustering of risk factors. Medicosocial workers attached to our department identified the residential area where most of them were insured persons of Employees State Insurance Act. Total 456 households were contacted and explained the importance of this study by conducting a meeting in their community hall premises.

- Procedure: Institutional ethics committee approval and written consent form from the participants were taken.
Data were collected by the trained team of community medicine for 5 days a week by visiting houses and interviewing the participants using a predesigned, pretested, and precoded questionnaire that took 30 minutes for each woman by the investigator. The interview schedule consisted of sociodemographic variables. Various risk factors such as dietary habits (using frequency questionnaire in which an intake of more than three times per week was considered as adequate), personal habits, lifestyle, physical activity, family history, any form of stress, number of sleep hours, reproductive parameters, anthropometry measurements height, weight, and waist circumference using standardized methods were taken. History of hypertension, diabetes, hyperlipidemia, thyroid disorders, and type of personality were noted. Questionnaire schedule was developed after a pilot test, and the necessary changes were incorporated.

A single blood pressure measurement was done by using standardized procedures in sitting position on the right arm using a digital sphygmomanometer (OMRON IAI) standardized every day with a diamond mercury sphygmomanometer. If found on the higher side, an average of three readings was taken with 10 minutes apart. Biochemical parameters such as fasting blood sugar and total cholesterol profile (after overnight fasting of 10-12 hours) using automated analyzer (Cobas C311) were measured at our hospital.

Framingham risk score that is validated and had been widely used to assess the 10-year risk of CVD was used. Absolute CVD risk percentage over 10 years was classified as low risk (<10\%), intermediate risk (10-20\%), and high risk (> 20\%). ${ }^{8}$ Those detected to be at intermediate risk were educated on primary prevention strategies such as lifestyle modifications and dietary changes, whereas those at high risk were referred to the noncommunicable disease clinic run at our urban health center, Jeedimetla, Hyderabad, for secondary prevention strategies such as early diagnosis and treatment. Also, if required, referral to our tertiary care hospital, Department of Cardiology, was also taken up. Clustering of atherogenic metabolic abnormalities is a major challenge among women and primary determinant of CVD. Metabolic syndrome was defined as per international diabetes federation. ${ }^{9}$

Statistical analysis: Data were entered and analyzed using SPSS v. 20 (IBM Inc.). Descriptive statistics such as percentages and mean \pm standard deviation; ANOVA (analysis of variance) was used to show the association of clustering of continuous variables (risk factors) with FRS categories in which three subgroups are present. Chi-square test was used to depict association between categorical and FRS subgroups ( $p<0.05$ taken as statistically significant value).

\section{Results}

Our study included 370 women who were unknown cases of coronary artery disease and stroke. Various risk factors were studied using WHO STEP that comprised three steps: behavioral, physical, and biochemical measurements. 
- Behavioral measurements included tobacco use, alcohol consumption, diet, physical activity, history of high raised blood pressure, and diabetes.

- Physical measurements included height, weight, waist circumference, and blood pressure.

- Biochemical measurements included blood glucose and blood lipids.

Apart from these stress, the number of hours of sleep and reproductive parameters were also assessed.

\section{Sociodemographic Characteristics of Study Patients}

Mean age of this study patients was found to be $34.21 \pm$ 12.4 years. The age-wise distribution is detailed in - Table 1 .

According to their religion, 324 (87.6\%) participants were Hindus, 25 (6.8\%) Muslims, and 21 (5.6\%) were Christians. As per their marital status, 267 (72.1\%) were married, 83 (22.5\%) were unmarried, 1 (0.3\%) was divorced, and 19 (5.1\%) were widows. Among these participants, 277 (74.9\%) belonged to the nuclear family, $80(21.6 \%)$ to joint family, and $13(3.5 \%)$ to extended family.

Educational status as classified constituted by 86 (23.3\%) illiterates and 284 (76.7\%) literates. Among these literates, graduates were 100 (27\%), postgraduates 14 (3.8\%), intermediate 54 (14.6\%), high school 66 (17.8\%), middle school 32 (8.6\%), and primary school were 18 (4.9\%).

The participants worked in various industries and contributed to 274 (74.1\%). Nonworking group constituted 96 (25.9\%). Among these workers, semiprofessional as supervisors or managers were $25(6.7 \%)$, clerical $26(7 \%)$, skilled 16 (4.3\%), semiskilled 127 (34.5\%), and unskilled were 80 (21.6\%).

Table 1 Age-wise distribution of study patients

\begin{tabular}{|l|l|l|}
\hline Age group $(\mathrm{y})$ & Number & Percentage \\
\hline $20-30$ & 195 & 53.3 \\
\hline $31-40$ & 74 & 20.2 \\
\hline $41-50$ & 52 & 14.2 \\
\hline $51-60$ & 34 & 9.3 \\
\hline$>60$ & 11 & 3 \\
\hline
\end{tabular}

Based on modified BG Prasad's classification, ${ }^{10} 84$ (22.7\%) participants belonged to the upper class, 165 (44.5\%) upper middle class, 83 (22.4\%) middle class, 27 (7.5\%) lower middle class, and 11 (2.9\%) belonged to the lower class (Fig. 1).

\section{Prevalence of Various Risk Factors among Study Participants: (Behavioral)}

Study participants consumed mixed diet catering to 344 (93\%) and vegetarian diet by only 26 (7\%). Diet habits were semiquantitatively measured by adopting food frequency questionnaire measured by their consumption of fiber-rich diet intake-greens more than three times a week 148 (40\%), less than three times a week 112 (30\%), never 110 (30\%); other vegetables more than three times a week 271 (73\%), less than three times a week $78(21 \%)$, never $21(6 \%)$; fruits more than three times a week $72(19 \%)$, less than three times a week 159 (43\%), never 139 (38\%). Saturated fat-rich diet intake-meat more than three times a week 33 (9\%), less than three times a week $241(65 \%)$, never 96 (26\%); poultry more than three times a week 85 (23\%), less than three times a week 241 (58\%), never 69 (19\%); deep-fried more than three times a week 65 (18\%), less than three times a week 178 (48\%), never 127 (34\%); junk food more than three times a week 38 (10\%), less than three times a week 152 (41\%), never 180 (49\%). High carbohydrate diet intake-high-starch foods more than three times a week 86 (24\%), less than three times a week 196 (53\%), never 88 (23\%); carbonated soft drinks more than three times a week $44(12 \%)$, less than three times a week 148 (40\%), never 178 (48\%). Extra salt intake in the form of table salt, papads, and chutneys were found among more than three times a week 98 (40\%), less than three times a week 168 (45\%), never 104 (28\%). Good cholesterol-rich food was consumed by only 14 (4\%) regularly.

Other behavioral factors: Alcohol usage was found among 22 (5.9\%) current users, 5 (1.4\%) past, and 343 (92.7\%) never; 25 (6.8\%); sedentary lifestyle 59 (16\%), and moderate 311 (84\%); extra physical activity 91 (25\%); history of hypertension 51 (14\%); and history of diabetes mellitus 34 (9.2\%).

Physical measurements: Waist circumference $>80 \mathrm{~cm}$ was found among 226 (61\%) women. Body mass index (BMI) as per

\section{Trend of metabolic syndrome as per age}

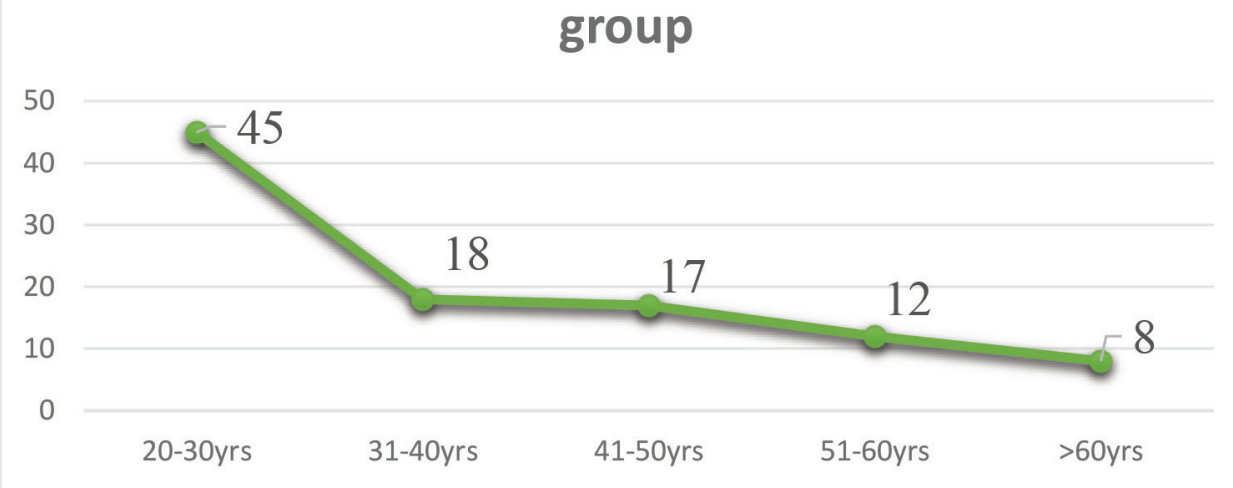

Fig. 1 Trend of metabolic syndrome as per age group. Chi-square test $=13.784 ; \mathrm{df}=4 ; p=0.008$. 
Asians classification-34 (9.1\%) were underweight, normal 107 (28.9\%), overweight 55 (14.8\%), and obese were 175 (47.2\%).

As per Joint National Committee 7 (JNC-7), prevalence of prehypertension was found among 46 (12.4\%) women; hypertension-I among 31 (8.4\%), and hypertension-II among $17(4.6 \%) .{ }^{11}$

Biochemical, metabolic factors: Raised blood glucose was found among 216 (58.4\%) women, raised triglycerides among 99 (26.7\%), and low HDL among 331 (89\%).

Other risk factors included a family history of hypertension or diabetes among 140 (38\%) women, any form of stress 140 (38\%), type A personality 42 (11\%), and sleep < 6 hours among 76 (22\%).

Specific risk factors for women were found with postmenopausal status among 70 (20\%) women and metabolic syndrome among 110 (29.7\%). Among these, it was found higher among younger age group (20-30 years) (-Fig. 1), history of gestational diabetes 7 (2\%), and pregnancy-induced hypertension among 0 (0\%). None of them had the hysterectomy.

\section{Categorization of Study Participants as per Framingham Risk Score}

As per FRS, low risk was among 347 (93.8\%) women, intermediate risk 14 (3.7\%), and high risk among 9 (2.5\%).

In ANOVA analysis, we found age, weight, waist circumference, systolic, diastolic, triglycerides, total cholesterol, and HDLs as significant factors with FRS (- Table 2)

In univariate analysis, nonworking, illiterates, upper middle class, postmenopausal status, and metabolic syndrome with FRS categorization were statistically significant ( - Table 3).

\section{Discussion}

Cross-sectional study was conducted in the industrial area of urbanized Hyderabad among 370 women to determine

Table 2 ANOVA analysis of various variables with FRS categorization

\begin{tabular}{|l|l|l|l|l|}
\hline Risk factors & Low risk & Intermediate risk & High risk & $p$ Value \\
\hline Age & $32.80+11.23$ & $57.14+9.3$ & $52.89+9.9$ & 0.0001 \\
\hline Weight & $55.76+13.5$ & $66.50+16.1$ & $61.00+6.36$ & 0.009 \\
\hline Waist circumference & $86.7+14.12$ & $106.07+12.70$ & $90.56+16.23$ & 0.0002 \\
\hline Systolic pressure & $115.65+15.34$ & $132.71+15.86$ & $132.89+21.35$ & 0.0004 \\
\hline Diastolic pressure & $77.52+9.34$ & $82.21+9.93$ & $87.56+10.6$ & 0.002 \\
\hline Triglycerides & $121.3+69.2$ & $195.5+41.98$ & $249.55+76$ & 0.0005 \\
\hline Total cholesterol & $156.83+42.9$ & $209.07+56.45$ & $258.33+94.24$ & 0.006 \\
\hline High-density lipoprotein & $39.36+10.4$ & $31.14+6.67$ & $33.11+24.4$ & 0.006 \\
\hline
\end{tabular}

Abbreviations: ANOVA, analysis of variance; FRS, Framingham risk score.

Table 3 Association of sociodemographic variables with Framingham risk score (FRS) categorization

\begin{tabular}{|c|c|c|c|c|c|}
\hline Variables & $\begin{array}{l}\text { Low } \\
N=347\end{array}$ & $\begin{array}{l}\text { Intermediate } \\
\mathrm{N}=14\end{array}$ & $\begin{array}{l}\text { High } \\
\mathrm{N}=9\end{array}$ & Chi-square df & $p$ Value \\
\hline \multicolumn{4}{|l|}{ Literacy status } & \multirow{3}{*}{$\begin{array}{l}24.236 \\
\mathrm{df}-2\end{array}$} & \multirow{3}{*}{0.003} \\
\hline Literate & $276(79.5 \%)$ & $5(35.7 \%)$ & $3(33.3)$ & & \\
\hline Illiterate & $71(20.5 \%)$ & $9(64.3 \%)$ & $6(66.7 \%)$ & & \\
\hline Working & $267(77 \%)$ & $1(7 \%)$ & $3(33 \%)$ & \multirow{2}{*}{$d f-2$} & \multirow{2}{*}{0.02} \\
\hline Non working & $80(23 \%)$ & $13(93 \%)$ & $6(67 \%)$ & & \\
\hline Upper class & $83(24 \%)$ & $1(7 \%)$ & $0(0 \%)$ & \multirow{5}{*}{$\begin{array}{l}27.8 \\
d f-4\end{array}$} & \multirow{5}{*}{0.001} \\
\hline Upper middle & $152(44 \%)$ & $10(72 \%)$ & $3(33 \%)$ & & \\
\hline Middle & $79(23 \%)$ & $1(7 \%)$ & $3(33 \%)$ & & \\
\hline Lower middle & $26(7 \%)$ & $0(0 \%)$ & $1(11 \%)$ & & \\
\hline Lower class & $7(2 \%)$ & $2(14 \%)$ & $2(23 \%)$ & & \\
\hline Pre menopause & $295(85 \%)$ & $2(14 \%)$ & $4(44 \%)$ & \multirow{2}{*}{$\mathrm{df}-2$} & \multirow{2}{*}{0.0004} \\
\hline Post menopause & $52(15 \%)$ & $12(86 \%)$ & $5(56 \%)$ & & \\
\hline \multicolumn{4}{|l|}{ Metabolic syndrome } & \multirow{3}{*}{$\begin{array}{l}57.96 \\
d f-2\end{array}$} & \multirow{3}{*}{0.0001} \\
\hline Yes & $260(75 \%)$ & $14(100 \%)$ & $9(100 \%)$ & & \\
\hline No & $87(25 \%)$ & $0(0 \%)$ & $0(0 \%)$ & & \\
\hline
\end{tabular}


various risk factors of CVDs using steps approach and specific risk factors to women. To estimate the risk over 10-year duration, FRS was used.

\section{Sociodemographic Characteristics of the Study Participants} Our study found that most women belonged to the age group of 20 to 30 years ( $53.3 \%$ ) and mean age was $34.21 \pm 12.4$ years. According to religion, majority (87.6\%) were Hindus and were married (72.1\%) with literacy status of $76.7 \%$. About $74 \%$ were in various industries as semiskilled workers predominantly belonging to upper middle class $44.5 \%$.

Contrast to our study, Mishra et al in their study conducted in urban slums of Cuttack city found only $30 \%$ literates, $66 \%$ married, and most of them belonged to lower class. ${ }^{12}$ Gupta et al also found that most of them belonged to lower middle class with the majority (52\%) of them between 40 and 49 years of age group. ${ }^{13}$ These variations could be explained by various settings in which this study was conducted.

\section{Prevalence of Various Risk Factors of Cardiovascular Diseases among Women of Industrial Area}

In our study of behavioral factors, most of them had a mixed diet (93\%). Regular intake of fruits was found among $19 \%$, green leafy vegetables $40 \%$, high saturated fat $9 \%$, junk and processed food $10 \%$; high carbohydrate/starch diet $24 \%$, carbonated soft drinks $12 \%$, and good cholesterol diet by only $4 \%$. Personal habits such as alcohol intake were there among $7.35 \%$ and tobacco consumption among $6.8 \%$, with sedentary lifestyle among $16 \%$. Extra physical activity in the form of walking, cycling, yoga, or meditation was present among $25 \%$; history of hypertension was present in $14 \%$ participants and diabetes in $9 \%$. Physical factors were found with marked overweight (14.8\%) and obesity (47.2\%). Waist circumference $>80 \mathrm{~cm}$ was observed among $67 \%$ of the participants. Prevalence of hypertension was detected at the time of interview among $13 \%$ using JNC-7. Biochemical parameters found raised blood glucose among $58.4 \%$ participants, raised triglycerides among $26.7 \%$, and low HDL among $89 \%$. Specific factors related to women were postmenopausal status among $20 \%$ participants and metabolic syndrome among $29.7 \%$; others included type A personality among $11 \%$ participants, stress $(28 \%)$, and sleep with $<6$ hours in $22 \%$.

Contrast to our study, the American Heart Stroke Association found that $13.6 \%$ of women were current smokers and $38 \%$ obese; raised cholesterol was found among $40.5 \%$ $\mathrm{NH}$ Asian women, $41.2 \%$ Hispanic women, 36\% black women, and $43.4 \%$ of white women. Diabetes mellitus was found among $9.1 \%$ of Asian women, $7.4 \%$ of white women, $13.6 \%$ of black women, and $12.7 \%$ of Hispanic women..$^{14}$ These big differences are mainly attributed to environmental conditions, lifestyle variations, and ethnicity.

Brazilian industrial workers study by Cassani et al on ANOVA found no significant variation of BMI for female sex. ${ }^{15}$ Islami et al in their prospective study of CVD and associated factors among Polish women found waist circumference and waist-hip ratio adjusted for diabetes mellitus and other factors association, which was statistically significant for highest quartile. ${ }^{16}$
The nationwide study by Pandey et al in India has found a higher prevalence of risk factors associated with CVDs among urban women. Usage of tobacco was found among $19.6 \%$ women, sedentary lifestyle $71 \%$, overweight $31.7 \%$, obese $13.9 \%$, waist circumference $>90 \mathrm{~cm}$, hypertension $48.2 \%$, diabetes $15 \%$, and hypercholesteremia $27.7 \% .{ }^{17}$

Gupta et al found low intake (less than three helpings per day) of fruits and vegetables among $23.6 \%$ women, smoking $4 \%$, smokeless tobacco usage $1.04 \%$, high-fat intake $31.3 \%$, sedentary physical activity $85.7 \%$, moderate (13\%); hypertensive (24.6\%); raised BMI > 25 among 50.7\% and $>30$ among $13.9 \%$; and waist circumference $>90 \mathrm{~cm} 54 \%$ of women. Raised triglycerides was found among $23 \%$, low HDL $43.7 \%$, and raised cholesterol among $47 \%{ }^{13}$ This could be due to various principles, different instruments involved for measuring, and different cutoff values opted by various researchers.

\section{Assessment of Cardiovascular Risk Estimate Using Framingham Risk Score}

In our study, the FRS estimated low risk among 93.4\%; intermediate risk among $3.7 \%$, and high risk among $2.5 \%$ over the next 10 years. On ANOVA, we found significant continuous variables such as increasing age, increasing weight, increasing waist circumference, both systolic and diastolic blood pressures, raised triglycerides, total cholesterol, and low HDL with FRS categorization. Univariate analysis using chi-square test was found to be statistically significant with increasing age, nonworking, illiterates, upper middle class, postmenopausal status, waist circumference $>80 \mathrm{~cm}$, triglycerides $>150 \mathrm{mg}$; HDL cholesterol $<50 \mathrm{mg} / \mathrm{dL}$, and metabolic syndrome.

Fatema et al, in their study of cardiovascular risk factors among Bangladeshi readymade garment workers, found $36 \%$ women with one intermediate risk factor, $29 \%$ with two intermediate risk factors, $12 \%$ with three intermediate risk factors, and $4 \%$ with four intermediate risk factors. They have also noted increased waist-hip ratio to be at high risk..$^{18}$

Parikh et al in their study to assess cardiovascular risk by using Framingham risk equation among urban residents of Ahmedabad city covering 1,210 population in different wards found low risk among $86.5 \%$, intermediate $9 \%$, and high risk among $4.5 \%$. This is in contrast to our study in which the increased risk was found with increased age group that is double between the age group of 55-59 and 60-64 years. Also, higher risk was among nonworking and those with inadequate sleep. ${ }^{19}$ These variations in the risk estimate could be due to different methods adopted to estimate.

\section{Prevalence of Metabolic Syndrome and a Major Determinant of Cardiovascular Disease}

Our study depicted $29.2 \%$ prevalence of metabolic syndrome that was found higher among 20 to 30 years of age group. This was statistically significant with FRS score in which prevalence was found more in intermediate and high risk.

Yousefzadeh et al in their population-based Kerman Coronary Artery Disease Risk study in Iran-in which metabolic syndrome was diagnosed as defined by the revised National Cholesterol Education Program definition criteria (ATPIII) and the FRS and was calculated using a computer 
program-previously reported algorithm found the prevalence of women with metabolic syndrome among $58.9 \%$. With FRS score, women with metabolic syndrome estimated of low risk were among $96.8 \%$, intermediate risk $3.2 \%$, and high risk $0.1 \%$. However, compared with nonmetabolic women, low risk was found in $99.7 \%$, intermediate risk in $0.3 \%$, and high risk in $0 \%$, which was statistically significant. In multiple logistic regression analysis, women with metabolic syndrome were at 12 times higher risk for intermediate risk estimate and 22 times for high risk. ${ }^{20}$ Tyagi et al in their study on biosocial predictors of hypertension among premenopausal and postmenopausal women found $29 \%$ of premenopausal women to be prehypertensive and $13.8 \%$ to be hypertensive. Among postmenopausal women, $26.2 \%$ were prehypertensive and $41 \%$ were hypertensive. In multinominal logistic regression analysis, the risk of each variable was found to be contributing to more than three times more likely to be hypertensive among postmenopausal women. Similarly, nonworking women had increased risk of developing blood pressure and 12 times greater obesity. ${ }^{21}$ Claudius et al in their study "Prevalence of Metabolic Syndrome among Women in Northern India" found among 300 women aged > 35 years, a higher prevalence of metabolic syndrome in $49.2 \%$ with increased age, parity, waist-hip ratio, and BMI. In logistic regression analysis, most significant predictor was BMI and menopausal status. ${ }^{22}$ Our study, in contrast, depicted increased metabolic syndrome among younger age group but similar increase among postmenopausal women. This could be due to the variation in their lifestyle and dietary habits that are predisposing to various components of metabolic syndrome.

One of the major limitations of our study is that we could not include the air pollutants as a risk factor of CVDs due to the availability constraints at the urban health center.

\section{Conclusion}

The highest prevalence of risk factors included the mainly poor intake of fruits, poor intake of good cholesterol diet (HDL) that protects from CVDs, excess salt intake, high central obesity, high blood glucose levels, and metabolic syndrome. FRS estimation, a simple, easily applicable method, was used to predict risk over 10 years among these women, which determined 3.7\% women at intermediate risk and 2.5\% at higher risk. This categorization was statistically significant among illiterates, nonworking group, increasing age, postmenopausal women, and women with metabolic syndrome that was found highest among the younger age group. Early screening strategies can be adopted, and these can be ameliorated at the primordial and primary level to prevent further progression.

\section{Conflict of Interest}

None.

\section{Funding}

Prajwalika Scholarship Program, Women in Cardiology Association.

\section{Acknowledgments}

We would extend our sincere thanks to Dr. Harshal Pandve, Head of the Department Community Medicine, Dr. A. Dhanalakshmi, Department of Biochemistry for her support in laboratory parameters, and Dr. Kamala, In-charge of ESIC Diagnostic Center, Jeedimetla, Hyderabad. We would thank the technician Venkatesh and medicosocial workers Shashanka, Shravan, and Paulson for extending their support. We would also thank all our study patients who gave their consent to participate in our project.

\section{References}

1 Clark H. NCDs: a challenge to sustainable human development. Lancet 2013;3819866:510-511

2 Andrade JP, Pinto FJ, Arnett DK. Prevention of Cardiovascular Diseases from Current Evidence to Clinical Practice. Cham, Switzerland: Springer International Publishing;2015:13-19

3 Prabhakaran D, Jeemon P, Sharma M. The changing patterns of cardiovascular diseases and their risk factors in the states of India: The Global Burden of Disease Study 1990-2016. India State-Level Disease Burden Initiative CVD Collaborators. Lancet Global Health2018. Published online September 12. Available at:http://www.thelancet.com/journal/langlo/ articlePIIS2214-109x(18)30407Accessed August 22, 2018>

4 The WHO STEP wise approach to Surveillance of non-communicable diseases (STEPS). Non-communicable Diseases and Mental Health. Geneva, Switzerland: World Health Organization;2003:17-20. Available at:http://www.who.int/ ncd_surveillanceAccessed August 10, 2015>

5 Towfighi A, Zheng L, Ovbiagele B. Sex-specific trends in midlife coronary heart disease risk and prevalence. Arch Intern Med 2009;169(19):1762-1766

6 Sohn C, Kim J, Bae W. The Framingham risk score, diet, and inflammatory markers in Korean men with metabolic syndrome. Nutr Res Pract 2012;6(3):246-253

7 Nag T, Ghosh A. Cardiovascular disease risk factors in Asian Indian population: a systematic review. J Cardiovasc Dis Res 2013;4(4):222-228

8 Kannel WB. Bishop lecture. Contribution of the Framingham Study to preventive cardiology. J Am Coll Cardiol 1990;15(1): 206-211

9 Alberti SG, Zimmet P, Shaw J, Grundy SM. The International Diabetic Federation consensus worldwide definition of the metabolic syndrome.2006:10. Available at:https://www.idf.org/ component/attachments/html. Accessed September 10, 2018>

10 Pandey VK, Aggarwal P, Kakkar R, Modified BG. Prasad's Socio-economic Classification-2018: the need of an update in the present scenario. Indian J Community Health 2018;30(1):82-84

11 Chobanian AV, Bakris GL, Black HR, et al; National Heart, Lung, and Blood Institute Joint National Committee on Prevention, Detection, Evaluation, and Treatment of High Blood Pressure; National High Blood Pressure Education Program Coordinating Committee. The Seventh Report of the Joint National Committee on Prevention, Detection, Evaluation, and Treatment of High Blood Pressure: the JNC 7 report. JAMA 2003;289(19):2560-2572

12 Mishra DK, Kar K, Panda M. Prevalence of risk factors for cardiovascular diseases among adults in slum area of Cuttack city, Odisha. Journal of Academia and Industrial Research. 2013;2(5):265-267 
13 Gupta E, Sharma KK, Gupta A, et al. Persistent High prevalence of cardiovascular risk factors in the urban middle class in India: Jaipur Heart Watch-5. J Assoc Phycians India 2012;60:11-16

14 Heart disease and stroke statistics2018. Update: a report from the American Heart Association. Circulation 2018; 137:e67-e492

15 Cassani RS, Nobre F, Filho AP, Schmidt A. Prevalence of cardiovascular risk factors in a population of Brazilian industry workers. Arq Bras Cardiol 2009;92(1):15-21

16 Islami F, Mańczuk M, Vedanthan R, et al. A cross-sectional study of cardiovascular disease and associated factors. Ann Agric Environ Med 2011;18(2):255-259

17 Pandey RM, Gupta R, Misra A, et al. Determinants of urban-rural differences in cardiovascular risk factors in middle-aged women in India: a cross-sectional study. Int J Cardiol 2013;163(2):157-162

18 Fatema K, Natasha K, Ali L. Cardiovascular risk factors among Bangladeshi ready-made garment workers. J Public Health Africa 2014;5(2):373
19 Parikh S, Patel M, Tiwari H, Bala DV, Joshi B. Assessment of cardiovascular disease risk by using Framingham risk equation amongst the residents of Ahmedabad city. Natl J Community Med 2013;4(3):392-397

20 Yousefzadeh G, Shokoohi M, Najafipour H, Shadkamfarokhi M. Applying the Framingham risk score for prediction of metabolic syndrome: the Kerman Coronary Artery Disease Risk Study, Iran. ARYA Atheroscler 2015;11(3):179-185

21 Tyagi R, Dhall M, Kapoor S. Bio-social predictors of hypertension among premenopausal and postmenopausal women. SAGE Open 2015; (January-March :1-12

22 Claudius E, Mandrelle K, John M, Singh S. Prevalence and predictors of metabolic syndrome among women above 35 years of cross-sectional study from northern India. Int J Reprod Contracept Obstet Gynecol 2016;5:1047-1051 